\title{
The Global Minimum of the Effective Potential of the Many-Electron System with Delta-Interaction
}

\author{
by \\ Detlef Lehmann* \\ Technische Universität Berlin \\ Fachbereich Mathematik Ma 7-2 \\ Staße des 17. Juni 136 \\ D-10623 Berlin, GERMANY
}

\begin{abstract}
We prove that the global minimum of the real part of the full effective potential of the many electron system with attractive delta interaction is in fact given by the BCS mean field configuration. This is a consequence of a simple bound which is obtained by applying Hadamard's inequality to the functional determinant. The second order Taylor expansion around the minimum is computed.
\end{abstract}

\footnotetext{
* e-mail: lehmann@math.tu-berlin.de
} 


\section{Introduction and Results}

In this article we consider the effective potential $V$ of the nonrelativistic many electron system with attractive delta function interaction. In momentum space, it reads

$$
\begin{aligned}
V\left(\left\{\phi_{q}\right\}\right) & =\sum_{q}\left|\phi_{q}\right|^{2}-\log \operatorname{det}\left[\begin{array}{cc}
I d & \frac{i g}{\sqrt{\beta L^{d}}} C \phi^{*} \\
\frac{i g}{\sqrt{\beta L^{d}}} \bar{C} \phi & I d
\end{array}\right] \\
& =\sum_{q}\left|\phi_{q}\right|^{2}-\log \operatorname{det}\left[I d+\frac{g^{2}}{\beta L^{d}} \bar{C} \phi C \phi^{*}\right]
\end{aligned}
$$

where $g^{2}=\lambda>0$ is an attractive coupling, $C=\left(\delta_{k, p} C_{k}\right)_{k, p}$ is a diagonal matrix with entries $C_{k}=\frac{1}{i k_{0}-\varepsilon_{\mathbf{k}}+\mu}, \varepsilon_{\mathbf{k}}=\varepsilon_{-\mathbf{k}}$ being the single particle energy momentum relation and $\mu$ denoting the chemical potential. In the following we set $e_{\mathbf{k}}=\varepsilon_{\mathbf{k}}-\mu$ such that the Fermi surface is given by $e_{\mathbf{k}}=0$. Furthermore $\phi$ is a short notation for the matrix $\left(\phi_{k-p}\right)_{k, p}$. The momenta $k=\left(k_{0}, \mathbf{k}\right), p=\left(p_{0}, \mathbf{p}\right)$ range over some finite subset of $\frac{\pi}{\beta}(2 \mathbf{Z}+1) \times\left(\frac{2 \pi}{L} \mathbf{Z}\right)^{d}$ if the system is kept in finite volume $[0, L]^{d}$ and at some small but positive temperature $T=\frac{1}{\beta}>0$. To be specific, choose

$$
k, p \in M_{\nu}:=\left\{\left(k_{0}, \mathbf{k}\right) \in \frac{\pi}{\beta}(2 \mathbf{Z}+1) \times\left(\frac{2 \pi}{L} \mathbf{Z}\right)^{d}|| e_{\mathbf{k}}|\leq 1,| k_{0} \mid \leq \nu\right\}
$$

where $1 \ll \nu<\infty$ is some cuttoff. The momenta $q=\left(q_{0}, \mathbf{q}\right)$ are given by $q \in\{k-p \mid k, p \in$ $\left.M_{\nu}\right\}$.

It is widely believed that the global minimum of the real part of $V$ (in general, the determinant in (1) is complex) is given by the mean field configuration $\phi_{x}=$ const in coordinate space or $\phi_{q} \sim \delta_{q, 0}$ in momentum space [1,2,3]. Although this is suggested by diagrammatic arguments [4], there was, to the authors knowledge, no rigorous proof of that. In Theorem 1 below we show that this result can be obtained by applying Hadamard's inequality (the absolute value of the determinant of $n$ column vectors is less than the volume of the cube spanned by the $n$ vectors) to the determinant in (1).

More precisely, all global minima of the real part

$$
\operatorname{Re} V\left(\left\{\phi_{q}\right\}\right)=\sum_{q}\left|\phi_{q}\right|^{2}-\log \left|\operatorname{det}\left[\begin{array}{cc}
I d & \frac{i g}{\sqrt{\beta L^{d}}} C \phi^{*} \\
\frac{i g}{\sqrt{\beta L^{d}}} \bar{C} \phi & I d
\end{array}\right]\right|
$$

of $V$ are given by

$$
\phi_{q}=\delta_{q, 0} \sqrt{\beta L^{d}} r_{0} e^{i \theta}
$$

where $\theta \in[0,2 \pi]$ is an arbitrary phase and $\Delta^{2}=\lambda r_{0}^{2}$ is a solution of the BCS equation

$$
\frac{\lambda}{\beta L^{d}} \sum_{k \in M_{\nu}} \frac{1}{k_{0}^{2}+e_{\mathbf{k}}^{2}+\lambda r_{0}^{2}}=1
$$


Equation (4) describes the BCS mean field configuration.

In Theorem 2 we expand $V$ up to second order in $\xi=\left(\xi_{k-p}\right)_{k, p}$ where

$$
\xi_{q}=\phi_{q}-\delta_{q, 0} \sqrt{\beta L^{d}} r_{0} e^{i \theta_{0}}= \begin{cases}\left(\rho_{0}-\sqrt{\beta L^{d}} r_{0}\right) e^{i \theta_{0}} & \text { for } q=0 \\ \rho_{q} e^{i \theta_{q}} & \text { for } q \neq 0\end{cases}
$$

We remark that a priori there is no need to introduce a small external field to fix the phase $\theta_{0}$ and then to expand with respect to radial and tangential components as it is usually done (for example $[2,3]$ ). The expansion around $\xi$ gives

$$
\begin{aligned}
V\left(\left\{\phi_{q}\right\}\right)= & V_{\min }+2 \beta_{0}\left(\rho_{0}-\sqrt{\beta L^{d}} r_{0}\right)^{2}+\sum_{q \neq 0}\left(\alpha_{q}+i \gamma_{q}\right) \rho_{q}^{2} \\
& +\frac{1}{2} \sum_{q \neq 0} \beta_{q}\left|e^{-i \theta_{0}} \phi_{q}+e^{i \theta_{0}} \bar{\phi}_{-q}\right|^{2}+O\left(\xi^{3}\right)
\end{aligned}
$$

where $V_{\min } \sim \beta L^{d} \Delta \log (1 / \Delta)$ is identical the global minimum of the BCS effective potential

$$
V_{\mathrm{BCS}}(\rho)=\beta L^{d}\left(\rho^{2}-\frac{1}{\beta L^{d}} \sum_{k} \log \left[1+\frac{\lambda \rho^{2}}{k_{0}^{2}+e_{\mathbf{k}}^{2}}\right]\right)
$$

and the coefficients $\alpha_{q}, \beta_{q}$ are real and positive and $\gamma_{q}$ is real. They are given in Theorem 2 below. In particular, $\alpha_{q} \sim q^{2}$ for small $q$.

The case where a small external U(1) symmetry breaking field is added to the fermionic action is discussed in section III.

The error term in (7) is not uniform in the coupling $\lambda$. Since $\beta_{q} \rightarrow 0$ and $\alpha_{q}+i \gamma_{q} \rightarrow 1$ for $\lambda \rightarrow 0$ (see (54)), equation (7) becomes in the limit $\lambda \rightarrow 0$

$$
\sum_{q}\left|\phi_{q}\right|^{2}=\sum_{q \neq 0}\left|\phi_{q}\right|^{2}+O\left(\xi^{3}\right)
$$

or $\left|\phi_{0}\right|^{2}=O\left(\xi^{3}\right)$. The reason for this can already be seen if one expands $V_{\mathrm{BCS}}$ above around its minimum $r_{0}$. One obtains, abbreviating $E_{k}^{2}:=k_{0}^{2}+e_{\mathbf{k}}^{2}+\Delta^{2}$,

$$
\begin{aligned}
\frac{1}{\beta L^{d}}\left(V_{\mathrm{BCS}}(\rho)-V_{\min }\right)=\rho^{2}-r_{0}^{2}-\frac{1}{\beta L^{d}} \sum_{k} \log \left[1+\frac{\lambda\left(\rho^{2}-r_{0}^{2}\right)}{k_{0}^{2}+e_{\mathbf{k}}^{2}+\Delta^{2}}\right] \\
=\left\{1-\frac{\lambda}{\beta L^{d}} \sum_{k} \frac{1}{E_{k}^{2}}\right\}\left(\rho^{2}-r_{0}^{2}\right)+\frac{1}{2} \frac{1}{\beta L^{d}} \sum_{k} \frac{\lambda^{2}}{E_{k}^{4}}\left(\rho^{2}-r_{0}^{2}\right)^{2}+O\left(\left(\rho-r_{0}\right)^{3}\right) \\
=\frac{1}{2} \frac{1}{\beta L^{d}} \sum_{k} \frac{\lambda^{2}}{E_{k}^{4}}\left[4 r_{0}^{2}\left(\rho-r_{0}\right)^{2}+4 r_{0}\left(\rho-r_{0}\right)^{3}+\left(\rho-r_{0}\right)^{4}\right]+O\left(\left(\rho-r_{0}\right)^{3}\right)
\end{aligned}
$$

where in the last line the BCS equation (5) has been used. Since $\frac{1}{\beta L^{d}} \sum_{k} \frac{1}{E_{k}^{4}} \sim \frac{1}{\Delta^{2}}=\frac{1}{\lambda r_{0}^{2}}$, the quadratic term on the right hand side of (10) goes to 0, whereas the third and fourth order terms diverge. 
Thus it is not clear to what extent one may draw conclusions from the quadratic approximation. Nevertheless, we write down the results for the partition function and the expectation value of the energy obtained by using (7). To this end we briefly recall the relation between the Hamiltonian and the functional integral representation of the model. One may look in [5] to see more detailed computations.

The Hamiltonian for the many-electron system with delta interaction in finite volume at some small but positive temperature $T=\frac{1}{\beta}>0$ described in the grand canonical ensemble is given by

$$
H=H_{0}-\lambda H_{\mathrm{int}}=\frac{1}{L^{d}} \sum_{\mathbf{k} \sigma}\left(\varepsilon_{\mathbf{k}}-\mu\right) a_{\mathbf{k} \sigma}^{+} a_{\mathbf{k} \sigma}-\frac{\lambda}{L^{3 d}} \sum_{\mathbf{k p q}} a_{\mathbf{k} \uparrow}^{+} a_{\mathbf{q}-\mathbf{k} \downarrow}^{+} a_{\mathbf{q}-\mathbf{p} \downarrow} a_{\mathbf{p} \uparrow}
$$

We are interested in the grand canonical partition function $\operatorname{Tr} e^{-\beta H}$, in the correlation function

$$
\tilde{\Lambda}(\mathbf{q})=\frac{1}{L^{3 d}} \sum_{\mathbf{k}, \mathbf{p}} \operatorname{Tr}\left[a_{\mathbf{k} \uparrow}^{+} a_{\mathbf{q}-\mathbf{k} \downarrow}^{+} a_{\mathbf{q}-\mathbf{p} \downarrow} a_{\mathbf{p} \uparrow} e^{-\beta H}\right] / \operatorname{Tr} e^{-\beta H}
$$

and in the expectation value $E_{\text {int }}=\left\langle H_{\text {int }}\right\rangle=\sum_{\mathbf{q}} \tilde{\Lambda}(\mathbf{q})$.

In terms of Grassmann integrals, the perturbation series for the normalized partition function $Z=\operatorname{Tr} e^{-\beta\left(H_{0}-\lambda H_{\mathrm{int}}\right)} / \operatorname{Tr} e^{-\beta H_{0}}$ is given by

$$
Z=\int e^{\frac{\lambda}{\left(\beta L^{d}\right)^{3}} \sum_{k, p, q} \bar{\psi}_{k \uparrow} \bar{\psi}_{q-k \downarrow} \psi_{q-p \downarrow} \psi_{p \uparrow}} d \mu_{C}
$$

and $\tilde{\Lambda}(\mathbf{q})$ is given by

$$
\tilde{\Lambda}(\mathbf{q})=\frac{1}{\beta} \sum_{q_{0} \in \frac{2 \pi}{\beta} \mathbf{Z}} \Lambda\left(q_{0}, \mathbf{q}\right)
$$

where

$$
\begin{aligned}
\Lambda\left(q_{0}, \mathbf{q}\right) & =\frac{1}{\left(\beta L^{d}\right)^{3}} \sum_{k, p}\left\langle\bar{\psi}_{k \uparrow} \bar{\psi}_{q-k \downarrow} \psi_{q-p \downarrow} \psi_{p \uparrow}\right\rangle \\
& =\frac{1}{\left(\beta L^{d}\right)^{3}} \sum_{k, p} \frac{1}{Z} \int \bar{\psi}_{k \uparrow} \bar{\psi}_{q-k \downarrow} \psi_{q-p \downarrow} \psi_{p \uparrow} e^{\frac{\lambda}{\left(\beta L^{d}\right)^{3}} \sum_{k, p, q} \bar{\psi}_{k \uparrow} \bar{\psi}_{q-k \downarrow} \psi_{q-p \downarrow} \psi_{p \uparrow}} d \mu_{C} \\
& =\left.\frac{d}{d \lambda_{q}}\right|_{\lambda_{q}=\lambda} \log \int e^{\frac{1}{\left(\beta L^{d}\right)^{3}} \sum_{k, p, q} \lambda_{q} \bar{\psi}_{k \uparrow} \bar{\psi}_{q-k \downarrow} \psi_{q-p \downarrow} \psi_{p \uparrow}} d \mu_{C}
\end{aligned}
$$

By making a Hubbard Stratonovich transformation, that is, by applying the formula $\left(\phi_{q}=\right.$ $\left.u_{q}+i v_{q}, \bar{\phi}_{q}=u_{q}-i v_{q}, d \phi_{q} d \bar{\phi}_{q}:=d u_{q} d v_{q}\right)$

$$
e^{\sum_{q} a_{q} b_{q}}=\int e^{\sum_{q} a_{q} \phi_{q}+b_{q} \bar{\phi}_{q}} e^{-\sum_{q}\left|\phi_{q}\right|^{2}} \prod_{q} \frac{d \phi_{q} d \bar{\phi}_{q}}{\pi}
$$


with $a_{q}=i g_{q}\left(\beta L^{d}\right)^{-\frac{3}{2}} \sum_{k} \bar{\psi}_{k \uparrow} \bar{\psi}_{q-k \downarrow}$ and $b_{q}=i g_{q}\left(\beta L^{d}\right)^{-\frac{3}{2}} \sum_{p} \psi_{p \uparrow} \psi_{q-p \downarrow}, g_{q}=\sqrt{\lambda}_{q}$, the exponent becomes quadratic in the Grassmann variables and the fermionic integral can be performed. Since the Grassmann variables in the exponent can be arranged, $\left(\bar{\psi}_{\uparrow}, \psi_{\downarrow}\right)$ and $\left(\psi_{\uparrow}, \bar{\psi}_{\downarrow}\right)$, such that a given $\psi$ appears only in one but not in both factors, the Pfaffian comming from the fermionic integration reduces to a determinant. The result is

$$
Z\left(\left\{\lambda_{q}\right\}\right)=\int e^{-V\left(\left\{\phi_{q}\right\}\right)} \prod_{q} \frac{d \phi_{q} d \bar{\phi}_{q}}{\pi}
$$

where, if the $\lambda_{q}$ 's are not all equal, instead of (1) $V$ is given by

$$
V\left(\left\{\phi_{q}\right\}\right)=\sum_{q}\left|\phi_{q}\right|^{2}-\log \operatorname{det}\left[I d+\frac{1}{\beta L^{d}} C(g \phi)^{*} \bar{C}(g \phi)\right]
$$

where $(g \phi)$ stands for the matrix $\left(g_{k-p} \phi_{k-p}\right)_{k, p}$. To perform the $\lambda_{q}$-derivative, one may change variables to obtain

$$
\begin{aligned}
\Lambda(q) & =\frac{1}{Z} \frac{d}{d \lambda_{q}} \int e^{-\sum_{q} \frac{\left|\phi_{q}\right|^{2}}{\lambda_{q}}} \operatorname{det}\left[I d+\frac{1}{\beta L^{d}} \bar{C} \phi C \phi^{*}\right] \Pi_{q} \frac{d \phi_{q} d \bar{\phi}_{q}}{\lambda_{q} \pi} \\
& =\frac{1}{Z} \int\left(\frac{\left|\phi_{q}\right|^{2}}{\lambda_{q}^{2}}-\frac{1}{\lambda_{q}}\right) e^{-\sum_{q} \frac{\left|\phi_{q}\right|^{2}}{\lambda}} \operatorname{det}\left[I d+\frac{1}{\beta L^{d}} \bar{C} \phi C \phi^{*}\right] \underset{q}{\prod_{q} \frac{d \phi_{q} d \bar{\phi}_{q}}{\lambda \pi}} \\
& =\frac{1}{Z} \int \frac{\left|\phi_{q}\right|^{2}-1}{\lambda} e^{-\sum_{q}\left|\phi_{q}\right|^{2}} \operatorname{det}\left[I d+\frac{\lambda}{\beta L^{d}} \bar{C} \phi C \phi^{*}\right] \prod_{q} \frac{d \phi_{q} d \bar{\phi}_{q}}{\pi} \\
& =\frac{1}{Z} \int \frac{\left|\phi_{q}\right|^{2}-1}{\lambda} e^{-V\left(\left\{\phi_{q}\right\}\right)} \prod_{q} \frac{d \phi_{q} d \bar{\phi}_{q}}{\pi}
\end{aligned}
$$

For the ideal Fermi gas $\lambda=0$ the above formula gives $\frac{0}{0}$. In that case one may use (15) to obtain

$$
\begin{aligned}
\Lambda(q) & =\frac{1}{\left(\beta L^{d}\right)^{3}} \sum_{k, p}\left\langle\bar{\psi}_{k \uparrow} \psi_{p \uparrow}\right\rangle\left\langle\bar{\psi}_{q-k \downarrow} \psi_{q-p \downarrow}\right\rangle=\frac{1}{\left(\beta L^{d}\right)^{3}} \sum_{k, p} \beta L^{d} \delta_{k, p} C_{k} \beta L^{d} \delta_{q-k, q-p} C_{q-k} \\
& =\frac{1}{\beta L^{d}} \sum_{k} C_{k} C_{q-k}
\end{aligned}
$$

which is the particle particle bubble.

If one replaces $V$ with the second order approximation

$$
V_{2}\left(\left\{\phi_{q}\right\}\right):=V_{\min }+2 \beta_{0}\left(\rho_{0}-\sqrt{\kappa} r_{0}\right)^{2}+\sum_{q \neq 0}\left(\alpha_{q}+i \gamma_{q}\right) \rho_{q}^{2}+\frac{1}{2} \sum_{q \neq 0} \beta_{q}\left|e^{-i \theta_{0}} \phi_{q}+e^{i \theta_{0}} \bar{\phi}_{-q}\right|^{2}
$$

the integrals in $(17,19)$ become Gaussian and can be performed. The results are (the index " 2 " in the following means that $V_{2}$ instead of $V$ has been used)

$$
Z_{2}=e^{-V_{\min }} \int_{0}^{\infty} e^{-2 \beta_{0}^{2}\left(\rho_{0}-\sqrt{\kappa} r_{0}\right)^{2}} 2 \rho_{0} d \rho_{0} \underset{\substack{q \\ q_{0}>0}}{\prod_{q} \frac{1}{\alpha_{q}^{2}+\gamma_{g}^{2}+2 \alpha_{q} \beta_{q}}}
$$




$$
\Lambda_{2}(q)=\frac{1}{\lambda}\left(\frac{\alpha_{q}+i \gamma_{q}+\beta_{q}}{\alpha_{q}^{2}+\gamma_{q}^{2}+2 \alpha_{q} \beta_{q}}-1\right)
$$

and, since $\gamma_{-q}=-\gamma_{q}$,

$$
\varepsilon_{\mathrm{int}, 2}:=\frac{1}{L^{d}}\left\langle H_{\mathrm{int}}\right\rangle_{2}=\frac{1}{\beta L^{d}} \sum_{q} \Lambda_{2}(q)=\frac{1}{\lambda} \frac{1}{\beta L^{d}} \sum_{q}\left(\frac{\alpha_{q}+\beta_{q}}{\alpha_{q}^{2}+\gamma_{q}^{2}+2 \alpha_{q} \beta_{q}}-1\right)
$$

In particular, since $\alpha_{q} \leq$ const $_{\lambda} q^{2}$ and $\left|\gamma_{q}\right| \leq$ const $_{\lambda}|q|$ for small $q$,

$$
\varepsilon_{\mathrm{int}, 2} \geq \frac{1}{\lambda} \frac{1}{\beta L^{d}} \sum_{q}\left(\frac{\text { const }_{\lambda}}{q^{2}}-1\right)
$$

which, for $L \rightarrow \infty$, is infrared singular for $d=1$ and, since $q_{0}=0$ is an allowed value for positive temperature, also logarithmically divergent for small $q$ in $d=2$. A similar observation is made in [3].

\section{Proof of Theorems}

Theorem 1: Let $\operatorname{Re} V$ be the real part of the effective potential for the many electron system with attractive delta interaction given by (3). Let $e_{\mathbf{k}}=\varepsilon_{\mathbf{k}}-\mu$ satisfy $e_{\mathbf{k}}=e_{-\mathbf{k}}$ and: $\forall \mathbf{k} e_{\mathbf{k}}=e_{\mathbf{k}+\mathbf{q}} \Rightarrow \mathbf{q}=0$. Then all global minima of ReV are given by (4),

$$
\phi_{q}=\delta_{q, 0} \sqrt{\beta L^{d}} r_{0} e^{i \theta}, \quad \theta \in[0,2 \pi] \text { arbitrary }
$$

where $r_{0}$ is a solution of the BCS equation (5) or, equivalently, the global minimum of the function (8)

$$
\begin{aligned}
V_{\mathrm{BCS}}(\rho) & =V\left(\left\{\delta_{q, 0} \sqrt{\beta L^{d}} \rho e^{i \theta}\right\}\right) \\
& =\beta L^{d} \rho^{2}-\sum_{k} \log \left[1+\frac{\lambda \rho^{2}}{k_{0}^{2}+e_{\mathbf{k}}^{2}}\right]=\beta L^{d} \rho^{2}-\sum_{\mathbf{k}} \log \left[\frac{\cosh \left(\frac{\beta}{2} \sqrt{e_{\mathbf{k}}^{2}+\lambda \rho^{2}}\right)}{\cosh \frac{\beta}{2} e_{\mathbf{k}}}\right]
\end{aligned}
$$

More specifically, there is the bound

$$
\begin{aligned}
& \operatorname{ReV}\left(\left\{\phi_{q}\right\}\right) \geq V_{\mathrm{BCS}}(\|\phi\|)-\min _{k \in M_{\nu}}\{\log {\left[\prod_{q \neq 0}\left(1-\frac{\left|\frac{\lambda}{\beta L^{d}} \sum_{p} \phi_{p} \bar{\phi}_{p+q}\right|^{2}}{\left(\left|a_{k}\right|^{2}+\lambda\|\phi\|^{2}\right)\left(\left|a_{k-q}\right|^{2}+\lambda\|\phi\|^{2}\right)}\right)^{\frac{1}{2}}\right] } \\
&\left.+\log \left[\prod_{q \neq 0}\left(1-\frac{\frac{\lambda}{\beta L^{d}}\left|\phi_{q}\right|^{2}\left|a_{k}-a_{k-q}\right|^{2}}{\left(\left|a_{k}\right|^{2}+\lambda|| \phi \|^{2}\right)\left(\left|a_{k-q}\right|^{2}+\lambda\|\phi\|^{2}\right)}\right)^{\frac{1}{2}}\right]\right\}
\end{aligned}
$$

where $\|\phi\|^{2}:=\frac{1}{\beta L^{d}} \sum_{q}\left|\phi_{q}\right|^{2}$ and $\left|a_{k}\right|^{2}:=k_{0}^{2}+e_{\mathbf{k}}^{2}$. In particular,

$$
\operatorname{Re} V\left(\left\{\phi_{q}\right\}\right) \geq V_{\mathrm{BCS}}(\|\phi\|)
$$


since the products in (26) are less or equal 1.

Proof: Suppose first that (26) holds. For each $q$, the round brackets in (26) are between 0 and 1 which means that $-\log \left(\Pi_{q} \cdots\right)$ is positive. Thus

$$
\operatorname{Re} V\left(\left\{\phi_{q}\right\}\right) \geq V_{\mathrm{BCS}}(\|\phi\|) \geq V_{\mathrm{BCS}}\left(r_{0}\right)
$$

which proves that $\phi_{q}=\delta_{q, 0} \sqrt{\beta L^{d}} r_{0} e^{i \theta}$ are indeed global minima of $\operatorname{Re} V$. On the other hand, if a configuration $\left\{\phi_{q}\right\}$ is a global minimum, then the logarithms in (26) must be zero for all $k \in M_{\nu}$ which in particular means that for all $q \neq 0$

$$
\sum_{p} \phi_{p} \bar{\phi}_{p+q}=0
$$

and for all $k \in M_{\nu}$ and $q \neq 0$

$$
\left|\phi_{q}\right|^{2}\left|a_{k}-a_{k-q}\right|^{2}=\left|\phi_{q}\right|^{2}\left[q_{0}^{2}+\left(e_{\mathbf{k}}-e_{\mathbf{k}-\mathbf{q}}\right)^{2}\right]=0
$$

which implies $\phi_{q}=0$ for all $q \neq 0$. It remains to prove (26).

To this end, we write (recall that $C_{k}=\frac{1}{a_{k}}=\frac{1}{i k_{0}-e_{\mathbf{k}}}$ )

$$
\operatorname{det}\left[\begin{array}{cc}
I d & \frac{i g}{\sqrt{\beta L^{d}}} \bar{C} \phi^{*} \\
\frac{i g}{\sqrt{\beta L^{d}}} C \phi & I d
\end{array}\right]=\operatorname{det}\left[\begin{array}{cc}
\mid & \mid \\
\vec{b}_{k} & \overrightarrow{b_{k^{\prime}}^{\prime}} \\
\mid & \mid
\end{array}\right]
$$

where ( $k, k^{\prime}$ fixed, $p$ labels the vector components)

$$
\vec{b}_{k}=\left(\begin{array}{c}
\delta_{k, p} \\
\frac{i g}{\sqrt{\beta L^{d}}} \frac{\phi_{k-p}}{a_{k}}
\end{array}\right) \quad \vec{b}_{k^{\prime}}^{\prime}=\left(\begin{array}{c}
\frac{i g}{\sqrt{\beta L^{d}}} \frac{\bar{\phi}_{p-k^{\prime}}}{\bar{a}_{k^{\prime}}} \\
\delta_{k^{\prime}, p}
\end{array}\right)
$$

If $\left|\vec{b}_{k}\right|$ denotes the euclidean norm of $\vec{b}_{k}$, then we have

$$
\left|\vec{b}_{k}\right|^{2}=1+\frac{\lambda}{\beta L^{d}} \sum_{p} \frac{\left|\phi_{k-p}\right|^{2}}{\left|a_{k}\right|^{2}}=1+\lambda \frac{\|\phi\|^{2}}{\left|a_{k}\right|^{2}}=\left|\vec{b}_{k}^{\prime}\right|^{2}
$$

Therefore one obtains, if $\vec{e}_{k}=\frac{\vec{b}_{k}}{\left|\vec{b}_{k}\right|}, \vec{e}_{k}^{\prime}=\frac{\vec{b}_{k}^{\prime}}{\left|\vec{b}_{k}^{\prime}\right|}$

$$
\operatorname{det}\left[\begin{array}{cc}
\mid & \mid \\
\vec{b}_{k} & \vec{b}_{k^{\prime}}^{\prime} \\
\mid & \mid
\end{array}\right]=\prod_{k}\left\{1+\lambda \frac{\|\phi\|^{2}}{\left|a_{k}\right|^{2}}\right\} \operatorname{det}\left[\begin{array}{cc}
\mid & \mid \\
\vec{e}_{k} & \vec{e}_{k^{\prime}}^{\prime} \\
\mid & \mid
\end{array}\right]
$$


From this the inequality (27) already follows since the determinant on the right hand side of (34) is less or equal 1. To obtain (26), we choose a fixed but arbitrary momentum $t \in M_{\nu}$ and orthogonalize all vectors $\vec{e}_{k}, \vec{e}_{k^{\prime}}^{\prime}$ in the determinant with respect to $e_{t}$. That is, we write

$$
\operatorname{det}\left[\begin{array}{ccc}
\mid & \mid & \mid \\
\vec{e}_{t} & \vec{e}_{k} & \vec{e}_{k^{\prime}}^{\prime} \\
\mid & \mid & \mid
\end{array}\right]=\operatorname{det}\left[\begin{array}{ccc}
\mid & \mid & \mid \\
\vec{e}_{t} & \vec{e}_{k}-\left(\vec{e}_{k}, \vec{e}_{t}\right) \vec{e}_{t} & \vec{e}_{k^{\prime}}^{\prime}-\left(\vec{e}_{k^{\prime}}^{\prime}, \vec{e}_{t}\right) \vec{e}_{t} \\
\mid & \mid & \mid
\end{array}\right]
$$

Finally we apply Hadamard's inequality, $|\operatorname{det} F| \leq \Pi_{j=1}^{n}\left|\vec{f}_{j}\right|=\left\{\Pi_{j=1}^{n} \sum_{i=1}^{n}\left|f_{i j}\right|^{2}\right\}^{\frac{1}{2}}$ if $F=\left(f_{i j}\right)_{1 \leq i, j \leq n}$ is a complex matrix, to the determinant on the right hand side of (35). Since

$$
\left|\vec{e}_{k}-\left(\vec{e}_{k}, \vec{e}_{t}\right) \vec{e}_{t}\right|^{2}=1-\left|\left(\vec{e}_{k}, \vec{e}_{t}\right)\right|^{2}
$$

one obtains

$$
\begin{aligned}
& \left|\operatorname{det}\left[\begin{array}{cc}
\vec{e}_{k}-\left(\vec{e}_{k}, \vec{e}_{t}\right) \vec{e}_{t} & \vec{e}_{k^{\prime}}^{\prime}-\left(\vec{e}_{k^{\prime}}^{\prime}, \vec{e}_{t}\right) \vec{e}_{t} \\
\mid & \mid
\end{array}\right]\right| \\
& \leq\left|\vec{e}_{t}\right| \prod_{\substack{k \in M_{\nu} \\
k \neq t}}\left(1-\left|\left(\vec{e}_{k}, \vec{e}_{t}\right)\right|^{2}\right)^{\frac{1}{2}} \prod_{k \in M_{\nu}}\left(1-\left|\left(\vec{e}_{k}^{\prime}, \vec{e}_{t}\right)\right|^{2}\right)^{\frac{1}{2}}
\end{aligned}
$$

or with (31) and (34)

$$
\begin{aligned}
\operatorname{Re} V\left(\left\{\phi_{q}\right\}\right) & =\sum_{q}\left|\phi_{q}\right|^{2}-\log \left|\operatorname{det}\left[\begin{array}{cc}
I d & \frac{i g}{\sqrt{\beta L^{d}}} \phi^{*} \bar{C} \\
\frac{i g}{\sqrt{\beta L^{d}}} \phi C & I d
\end{array}\right]\right| \\
& =\beta L^{d}\|\phi\|^{2}-\sum_{k} \log \left\{1+\lambda \frac{\|\phi\|^{2}}{\left|a_{k}\right|^{2}}\right\}-\log \left|\operatorname{det}\left[\begin{array}{cc}
\mid & \mid \\
\vec{e}_{k} & \vec{e}_{k^{\prime}}^{\prime} \\
\mid & \mid
\end{array}\right]\right| \\
& =V_{\mathrm{BCS}}(\|\phi\|)-\log \left|\operatorname{det}\left[\begin{array}{cc}
\mid & \mid \\
\vec{e}_{k} & \vec{e}_{k^{\prime}}^{\prime} \\
\mid & \mid
\end{array}\right]\right| \\
& \geq V_{\mathrm{BCS}}(\|\phi\|)-\log \left\{\prod_{\substack{k \in M_{\nu} \\
k \neq t}}\left(1-\left|\left(\vec{e}_{k}, \vec{e}_{t}\right)\right|^{2}\right)^{\frac{1}{2}} \underset{k \in M_{\nu}}{\left.\prod_{k \in M_{\nu}}\left(1-\left|\left(\vec{e}_{k}^{\prime}, \vec{e}_{t}\right)\right|^{2}\right)^{\frac{1}{2}}\right\}}\right.
\end{aligned}
$$

Finally one has

$$
\left(\vec{b}_{k}, \vec{b}_{t}\right)=\sum_{p} \frac{i g}{\sqrt{\beta L^{d}}} \frac{\phi_{k-p}}{a_{k}} \overline{\frac{i g}{\sqrt{\beta L^{d}}} \frac{\phi_{t-p}}{a_{t}}}
$$

which gives

$$
\left|\left(\vec{e}_{k}, \vec{e}_{t}\right)\right|^{2}=\frac{\left|\frac{\lambda}{\beta L^{d}} \sum_{p} \phi_{k-p} \bar{\phi}_{t-p}\right|^{2}}{\left(\left|a_{k}\right|^{2}+\lambda\|\phi\|^{2}\right)\left(\left|a_{t}\right|^{2}+\lambda\|\phi\|^{2}\right)}=\frac{\left|\frac{\lambda}{\beta L^{d}} \sum_{p} \phi_{p} \bar{\phi}_{t-k+p}\right|^{2}}{\left(\left|a_{k}\right|^{2}+\lambda\|\phi\|^{2}\right)\left(\left|a_{t}\right|^{2}+\lambda\|\phi\|^{2}\right)}
$$


and

$$
\left(\vec{b}_{k}^{\prime}, \vec{b}_{t}\right)=\frac{i g}{\sqrt{\beta L^{d}}} \frac{\bar{\phi}_{t-k}}{\bar{a}_{k}}+\overline{\frac{i g}{\sqrt{\beta L^{d}}} \frac{\phi_{t-k}}{a_{t}}}=\frac{i g}{\sqrt{\beta L^{d}}} \bar{\phi}_{t-k}\left(\frac{1}{\bar{a}_{k}}-\frac{1}{\bar{a}_{t}}\right)
$$

which gives

$$
\left|\left(\vec{e}_{k}^{\prime}, \vec{e}_{t}\right)\right|^{2}=\frac{\frac{\lambda}{\beta L^{d}}\left|\phi_{t-k}\right|^{2}\left|a_{t}-a_{k}\right|^{2}}{\left(\left|a_{k}\right|^{2}+\lambda\|\phi\|^{2}\right)\left(\left|a_{t}\right|^{2}+\lambda\|\phi\|^{2}\right)}
$$

Substituting $(38 \mathrm{a}, \mathrm{b})$ in (37) gives, substituting $k \rightarrow q=t-k$

$$
\begin{aligned}
\operatorname{Re} V\left(\left\{\phi_{q}\right\}\right) & \geq V_{\mathrm{BCS}}(\|\phi\|)-\log \left\{\prod_{\substack{k \in M_{\nu} \\
k \neq t}}\left(1-\left|\left(\vec{e}_{k}, \vec{e}_{t}\right)\right|^{2}\right)^{\frac{1}{2}} \prod_{k \in M_{\nu}}\left(1-\left|\left(\vec{e}_{k}^{\prime}, \vec{e}_{t}\right)\right|^{2}\right)^{\frac{1}{2}}\right\} \\
& \stackrel{k=t-q}{=} V_{\mathrm{BCS}}(\|\phi\|)-\log \left\{\prod_{q \neq 0}\left(1-\left|\left(\vec{e}_{t-q}, \vec{e}_{t}\right)\right|^{2}\right)^{\frac{1}{2}} \prod_{q \neq 0}\left(1-\left|\left(\vec{e}_{t-q}^{\prime}, \vec{e}_{t}\right)\right|^{2}\right)^{\frac{1}{2}}\right\}
\end{aligned}
$$

Since $t$ was arbitrary, we can take the maximum of the right hand side of (39) with respect to $t$ which proves Theorem 1

Theorem 2: Let $V$ be the effective potential (1), let $\kappa=\beta L^{d}$ and let $\xi=\left(\xi_{k-p}\right)_{k, p}$ be the matrix with entries

$$
\xi_{q}=\phi_{q}-\delta_{q, 0} \sqrt{\kappa} r_{0} e^{i \theta_{0}}= \begin{cases}\left(\rho_{0}-\sqrt{\kappa} r_{0}\right) e^{i \theta_{0}} & \text { for } q=0 \\ \rho_{q} e^{i \theta_{q}} & \text { for } q \neq 0\end{cases}
$$

Then

$V\left(\left\{\phi_{q}\right\}\right)=V_{\min }+2 \beta_{0}\left(\rho_{0}-\sqrt{\kappa} r_{0}\right)^{2}+\sum_{q \neq 0}\left(\alpha_{q}+i \gamma_{q}\right) \rho_{q}^{2}+\frac{1}{2} \sum_{q \neq 0} \beta_{q}\left|e^{-i \theta_{0}} \phi_{q}+e^{i \theta_{0}} \bar{\phi}_{-q}\right|^{2}+O\left(\xi^{3}\right)$

where, if $E_{k}^{2}=k_{0}^{2}+e_{\mathbf{k}}^{2}+\lambda r_{0}^{2}$,

$$
\begin{gathered}
\alpha_{q}=\frac{1}{2} \frac{\lambda}{\kappa} \sum_{k} \frac{q_{0}^{2}+\left(e_{\mathbf{k}}-e_{\mathbf{k}-\mathbf{q}}\right)^{2}}{E_{k}^{2} E_{k-q}^{2}}>0, \quad \beta_{q}=\frac{\lambda}{\kappa} \sum_{k} \frac{\lambda r_{0}^{2}}{E_{k}^{2} E_{k-q}^{2}}>0 \\
\gamma_{q}=-\frac{\lambda}{\kappa} \sum_{k} \frac{k_{0} e_{\mathbf{k}-\mathbf{q}}-\left(k_{0}-q_{0}\right) e_{\mathbf{k}}}{E_{k}^{2} E_{k-q}^{2}} \in \mathbf{R}
\end{gathered}
$$

and

$$
V_{\min }=\kappa\left(r_{0}^{2}-\frac{1}{\kappa} \sum_{\mathbf{k}} \log \left[\frac{\cosh \left(\frac{\beta}{2} \sqrt{e_{\mathbf{k}}^{2}+\lambda r_{0}^{2}}\right)}{\cosh \frac{\beta}{2} e_{\mathbf{k}}}\right]\right)
$$


Proof: We abbreviate $\kappa=\beta L^{d}$ and write

$$
V\left(\left\{\phi_{q}\right\}\right)=\sum_{q}\left|\phi_{q}\right|^{2}-\log \frac{\operatorname{det}\left[\begin{array}{cc}
A & \frac{i g}{\sqrt{\kappa}} \phi^{*} \\
\frac{i g}{\sqrt{\kappa}} \phi & \bar{A}
\end{array}\right]}{\operatorname{det}\left[\begin{array}{cc}
A & 0 \\
0 & \bar{A}
\end{array}\right]}
$$

where $A=C^{-1}=\left(\delta_{k, p} a_{k}\right)_{k, p \in M_{\kappa}}$ and $a_{k}:=1 / C_{k}=i k_{0}-e_{\mathbf{k}}$. Then

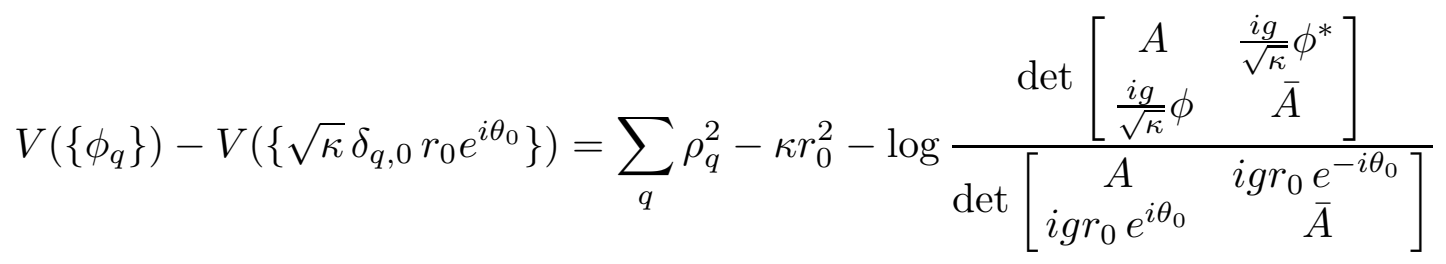

where $i g r_{0} e^{i \theta_{0}} \equiv i g r_{0} e^{i \theta_{0}} I d$ in the determinant above. Since

$$
\begin{aligned}
{\left[\begin{array}{cc}
A & i g r_{0} e^{-i \theta_{0}} \\
i g r_{0} e^{i \theta_{0}} & \bar{A}
\end{array}\right]^{-1} } & =\left[\begin{array}{cc}
\frac{\bar{a}_{k} \delta_{k, p}}{\left|a_{k}\right|^{2}+\lambda r_{0}^{2}} & -\frac{i g r_{0} e^{-i \theta_{0}} \delta_{k, p}}{\left|a_{k}\right|^{2}+\lambda r_{0}^{2}} \\
-\frac{i g r_{0} e^{i \theta_{0} \delta_{k, p}}}{\left|a_{k}\right|^{2}+\lambda r_{0}^{2}} & \frac{a_{k} \delta_{k, p}}{\left|a_{k}\right|^{2}+\lambda r_{0}^{2}}
\end{array}\right] \\
& \equiv \frac{1}{|a|^{2}+\lambda r_{0}^{2}}\left[\begin{array}{cc}
\bar{A} & -i g r_{0} e^{-i \theta_{0}} \\
-i g r_{0} e^{i \theta_{0}} & A
\end{array}\right]
\end{aligned}
$$

and because of

$$
\begin{aligned}
{\left[\begin{array}{cc}
A & \frac{i g}{\sqrt{\kappa}} \phi^{*} \\
\frac{i g}{\sqrt{\kappa}} \phi & \bar{A}
\end{array}\right] } & =\left[\begin{array}{cc}
A & i g r_{0} e^{-i \theta_{0}} \\
i g r_{0} e^{i \theta_{0}} & \bar{A}
\end{array}\right]+\left[\begin{array}{cc}
0 & \frac{i g}{\sqrt{\kappa} \phi^{*}}-i g r_{0} e^{-i \theta_{0}} \\
\frac{i g}{\sqrt{\kappa} \phi}-i g r_{0} e^{i \theta_{0}} & 0
\end{array}\right] \\
& =\left[\begin{array}{cc}
A & i g \bar{\gamma} \\
i g \gamma & \bar{A}
\end{array}\right]+\left[\begin{array}{cc}
0 & i g \xi^{*} \\
i g \xi & 0
\end{array}\right]
\end{aligned}
$$

where $\gamma=r_{0} e^{i \theta_{0}}$ and $\xi=\left(\xi_{k, p}\right)$ is given by (40), the quotient of determinants in (45) is given by

$$
\begin{aligned}
\operatorname{det}\left[I d+\frac{1}{|a|^{2}+\lambda r_{0}^{2}}\right. & \left.\left(\begin{array}{cc}
\bar{A} & -i g \bar{\gamma} \\
-i g \gamma & A
\end{array}\right)\left(\begin{array}{cc}
0 & i g \xi^{*} \\
i g \xi & 0
\end{array}\right)\right] \\
& =\operatorname{det}\left[I d+\left(\begin{array}{cc}
\lambda \frac{\bar{\gamma}}{|a|^{2}+\lambda r_{0}^{2}} \xi & i g \frac{\bar{A}}{|a|^{2}+\lambda r_{0}^{2}} \xi^{*} \\
i g \frac{A}{|a|^{2}+\lambda r_{0}^{2}} \xi & \lambda \frac{\gamma}{|a|^{2}+\lambda r_{0}^{2}} \xi^{*}
\end{array}\right)\right]
\end{aligned}
$$

Since

$$
\begin{aligned}
\log \operatorname{det}[I d+B] & =\operatorname{Tr} \log [I d+B]=\sum_{n=1}^{\infty} \frac{(-1)^{n+1}}{n} \operatorname{Tr} B^{n} \\
& =\operatorname{Tr} B-\frac{1}{2} \operatorname{Tr} B^{2}+\frac{1}{3} \operatorname{Tr} B^{3}-+\cdots
\end{aligned}
$$


one obtains to second order in $\xi$ :

$$
\begin{aligned}
\log \operatorname{det} & {\left[I d+\left(\begin{array}{cc}
\lambda \frac{\bar{\gamma}}{|a|^{2}+\lambda r_{0}^{2}} \xi & i g \frac{\bar{A}}{\overline{|a|^{2}+\lambda r_{0}^{2}}} \xi^{*} \\
i g \frac{A}{|a|^{2}+\lambda r_{0}^{2}} \xi & \lambda \frac{\gamma}{|a|^{2}+\lambda r_{0}^{2}} \xi^{*}
\end{array}\right)\right] } \\
= & \operatorname{Tr}\left(\begin{array}{cc}
\lambda \frac{\bar{\gamma}}{|a|^{2}+\lambda r_{0}^{2}} \xi & i g \frac{\bar{A}}{|a|^{2}+\lambda r_{0}^{2}} \xi^{*} \\
i g \frac{A}{|a|^{2}+\lambda r_{0}^{2}} \xi & \lambda \frac{\gamma}{|a|^{2}+\lambda r_{0}^{2}} \xi^{*}
\end{array}\right)-\frac{1}{2} \operatorname{Tr}\left\{\left(\begin{array}{cc}
\lambda \frac{\bar{\gamma}}{|a|^{2}+\lambda r_{0}^{2}} \xi & i g \frac{\bar{A}}{|a|^{2}+\lambda r_{0}^{2}} \xi^{*} \\
i g \frac{A}{|a|^{2}+\lambda r_{0}^{2}} \xi & \lambda \frac{\gamma}{|a|^{2}+\lambda r_{0}^{2}} \xi^{*}
\end{array}\right)^{2}\right\}+O\left(\xi^{3}\right) \\
= & \operatorname{Tr} \frac{\lambda \bar{\gamma}}{|a|^{2}+\lambda r_{0}^{2}} \xi+\operatorname{Tr} \frac{\lambda \gamma}{|a|^{2}+\lambda r_{0}^{2}} \xi^{*}-\frac{1}{2}\left\{\operatorname{Tr} \frac{\lambda \bar{\gamma}}{|a|^{2}+\lambda r_{0}^{2}} \xi \frac{\lambda \bar{\gamma}}{|a|^{2}+\lambda r_{0}^{2}} \xi+\operatorname{Tr} \frac{i g \bar{A}}{|a|^{2}+\lambda r_{0}^{2}} \xi^{*} \frac{i g A}{|a|^{2}+\lambda r_{0}^{2}} \xi\right. \\
& \left.+\operatorname{Tr} \frac{\lambda \gamma}{|a|^{2}+\lambda r_{0}^{2}} \xi^{*} \frac{\lambda \gamma}{|a|^{2}+\lambda r_{0}^{2}} \xi^{*}+\operatorname{Tr} \frac{i g A}{|a|^{2}+\lambda r_{0}^{2}} \xi \frac{i g \bar{A}}{|a|^{2}+\lambda r_{0}^{2}} \xi^{*}\right\}+O\left(\xi^{3}\right)
\end{aligned}
$$

One has

$$
\begin{aligned}
\left(\frac{\lambda \bar{\gamma}}{|a|^{2}+\lambda r_{0}^{2}} \xi\right)_{k, p}= & \frac{\lambda \bar{\gamma}}{\left.|a|_{k}\right|^{2}+\lambda r_{0}^{2}} \xi_{k, p}, \quad\left(\frac{\lambda \gamma}{|a|^{2}+\lambda r_{0}^{2}} \xi^{*}\right)_{k, p}=\frac{\lambda \gamma}{\left|a_{k}\right|^{2}+\lambda r_{0}^{2}} \bar{\xi}_{p, k} \\
\left(\frac{\lambda \bar{\gamma}}{|a|^{2}+\lambda r_{0}^{2}} \xi \frac{\lambda \bar{\gamma}}{|a|^{2}+\lambda r_{0}^{2}} \xi\right)_{k, k} & =\sum_{p} \frac{\lambda \bar{\gamma}}{\left|a_{k}\right|^{2}+\lambda r_{0}^{2}} \xi_{k, p} \frac{\lambda \bar{\gamma}}{\left|a_{p}\right|^{2}+\lambda r_{0}^{2}} \xi_{p, k} \\
& =\left(\frac{\lambda \bar{\gamma} \xi_{k, k}}{\left|a_{k}\right|^{2}+\lambda r_{0}^{2}}\right)^{2}+\sum_{\substack{p \\
p \neq k}} \frac{\lambda \bar{\gamma}}{\left|a_{k}\right|^{2}+\lambda r_{0}^{2}} \frac{\lambda \bar{\gamma}}{\left|a_{p}\right|^{2}+\lambda r_{0}^{2}} \xi_{k, p} \xi_{p, k} \\
& =\frac{1}{\kappa}\left(\frac{\lambda r_{0}\left(\rho_{0}-\sqrt{\kappa} r_{0}\right)}{\left|a_{k}\right|^{2}+\lambda r_{0}^{2}}\right)^{2}+\frac{1}{\kappa} \sum_{q \neq 0} \frac{\lambda \bar{\gamma}}{\left|a_{k}\right|^{2}+\lambda r_{0}^{2}} \frac{\lambda \bar{\gamma}}{\left|a_{k-q}\right|^{2}+\lambda r_{0}^{2}} \phi_{q} \phi_{-q} \\
\left(\frac{\lambda \gamma}{|a|^{2}+\lambda r_{0}^{2}} \xi^{*} \frac{\lambda \gamma}{|a|^{2}+\lambda r_{0}^{2}} \xi^{*}\right)_{k, k} & =\sum_{p} \frac{\lambda \gamma}{\left|a_{k}\right|^{2}+\lambda r_{0}^{2}} \bar{\xi}_{p, k} \frac{\lambda \gamma}{\left|a_{p}\right|^{2}+\lambda r_{0}^{2}} \bar{\xi}_{k, p} \\
& =\left(\frac{\lambda \gamma \bar{\xi}_{k, k}}{\left|a_{k}\right|^{2}+\lambda r_{0}^{2}}\right)^{2}+\sum_{\substack{p \\
p \neq k}} \frac{\lambda \gamma}{\left|a_{k}\right|^{2}+\lambda r_{0}^{2}} \frac{\lambda \gamma}{\left|a_{p}\right|^{2}+\lambda r_{0}^{2}} \bar{\xi}_{k, p} \bar{\xi}_{p, k} \\
& =\frac{1}{\kappa}\left(\frac{\lambda r_{0}\left(\rho_{0}-\sqrt{\kappa} r_{0}\right)}{\left|a_{k}\right|^{2}+\lambda r_{0}^{2}}\right)^{2}+\frac{1}{\kappa} \sum_{q \neq 0} \frac{\lambda \gamma}{\left|a_{k}\right|^{2}+\lambda r_{0}^{2}} \frac{\lambda \gamma}{\left|a_{k-q}\right|^{2}+\lambda r_{0}^{2}} \bar{\phi}_{q} \bar{\phi}_{-q}
\end{aligned}
$$

and

$$
\begin{aligned}
\left(\frac{i g A}{|a|^{2}+\lambda r_{0}^{2}} \xi\right)_{k, p} & =\frac{i g a_{k}}{\left|a_{k}\right|^{2}+\lambda r_{0}^{2}} \xi_{k, p}, \quad\left(\frac{i g \bar{A}}{|a|^{2}+\lambda r_{0}^{2}} \xi^{*}\right)_{k, p}=\frac{i g \bar{a}_{k}}{\left|a_{k}\right|^{2}+\lambda r_{0}^{2}} \bar{\xi}_{p, k} \\
\left(\frac{i g A}{|a|^{2}+\lambda r_{0}^{2}} \xi \frac{i g \bar{A}}{|a|^{2}+\lambda r_{0}^{2}} \xi^{*}\right)_{k, k} & =\sum_{p} \frac{i g a_{k}}{\left|a_{k}\right|^{2}+\lambda r_{0}^{2}} \xi_{k, p} \frac{i g \bar{a}_{p}}{\left|a_{p}\right|^{2}+\lambda r_{0}^{2}} \bar{\xi}_{k, p} \\
& =-\lambda \frac{\left|a_{k}\right|^{2}}{\left(\left|a_{k}\right|^{2}+\lambda r_{0}^{2}\right)^{2}}\left|\xi_{k, k}\right|^{2}-\lambda \sum_{\substack{p \\
p \neq k}} \frac{a_{k}}{\left|a_{k}\right|^{2}+\lambda r_{0}^{2}} \xi_{k, p} \frac{\bar{a}_{p}}{\left|a_{p}\right|^{2}+\lambda r_{0}^{2}} \bar{\xi}_{k, p} \\
& =-\frac{\lambda}{\kappa} \frac{\left|a_{k}\right|^{2}}{\left(\left|a_{k}\right|^{2}+\lambda r_{0}^{2}\right)^{2}}\left(\rho_{0}-\sqrt{\kappa} r_{0}\right)^{2}-\frac{\lambda}{\kappa} \sum_{q \neq 0} \frac{a_{k}}{\left|a_{k}\right|^{2}+\lambda r_{0}^{2}} \frac{\bar{a}_{k-q}}{\left|a_{k-q}\right|^{2}+\lambda r_{0}^{2}} \rho_{q}^{2}
\end{aligned}
$$




$$
\begin{aligned}
\left(\frac{i g \bar{A}}{|a|^{2}+\lambda r_{0}^{2}} \xi^{*} \frac{i g A}{|a|^{2}+\lambda r_{0}^{2}} \xi\right)_{k, k} & =\sum_{p} \frac{i g \bar{a}_{k}}{\left|a_{k}\right|^{2}+\lambda r_{0}^{2}} \bar{\xi}_{p, k} \frac{i g a_{p}}{\left|a_{p}\right|^{2}+\lambda r_{0}^{2}} \xi_{p, k} \\
& =-\lambda \frac{\left|a_{k}\right|^{2}}{\left(\left|a_{k}\right|^{2}+\lambda r_{0}^{2}\right)^{2}}\left|\xi_{k, k}\right|^{2}-\lambda \sum_{p} \frac{\bar{a}_{k}}{\left|a_{k}\right|^{2}+\lambda r_{0}^{2}} \bar{\xi}_{p, k} \frac{a_{p}}{\left|a_{p}\right|^{2}+\lambda r_{0}^{2}} \xi_{p, k} \\
& =-\frac{\lambda}{\kappa} \frac{\left|a_{k}\right|^{2}}{\left(\left|a_{k}\right|^{2}+\lambda r_{0}^{2}\right)^{2}}\left(\rho_{0}-\sqrt{\kappa} r_{0}\right)^{2}-\frac{\lambda}{\kappa} \sum_{q \neq 0} \frac{\bar{a}_{k}}{\left|a_{k}\right|^{2}+\lambda r_{0}^{2}} \frac{a_{k+q}}{\left|a_{k+q}\right|^{2}+\lambda r_{0}^{2}} \rho_{q}^{2}
\end{aligned}
$$

Therefore (50) becomes

$$
\begin{aligned}
\log \operatorname{det} & {\left[I d+\left(\begin{array}{cc}
\lambda \frac{\bar{\gamma}}{|a|^{2}+\lambda r_{0}^{2}} \xi & i g \frac{\bar{A}}{|a|^{2}+\lambda r_{0}^{2}} \xi^{*} \\
i g \frac{A}{|a|^{2}+\lambda r_{0}^{2}} \xi & \lambda \frac{\gamma}{|a|^{2}+\lambda r_{0}^{2}} \xi^{*}
\end{array}\right)\right] } \\
= & \operatorname{Tr} \frac{\lambda \bar{\gamma}}{|a|^{2}+\lambda r_{0}^{2}} \xi+\operatorname{Tr} \frac{\lambda \gamma}{|a|^{2}+\lambda r_{0}^{2}} \xi^{*}-\frac{1}{2}\left\{\operatorname{Tr} \frac{\lambda \bar{\gamma}}{|a|^{2}+\lambda r_{0}^{2}} \xi \frac{\lambda \bar{\gamma}}{|a|^{2}+\lambda r_{0}^{2}} \xi+\operatorname{Tr} \frac{i g \bar{A}}{|a|^{2}+\lambda r_{0}^{2}} \xi^{*} \frac{i g A}{|a|^{2}+\lambda r_{0}^{2}} \xi\right. \\
& \left.+\operatorname{Tr} \frac{\lambda \gamma}{|a|^{2}+\lambda r_{0}^{2}} \xi^{*} \frac{\lambda \gamma}{|a|^{2}+\lambda r_{0}^{2}} \xi^{*}+\operatorname{Tr} \frac{i g A}{|a|^{2}+\lambda r_{0}^{2}} \xi \frac{i g \bar{A}}{|a|^{2}+\lambda r_{0}^{2}} \xi^{*}\right\}+O\left(\xi^{3}\right) \\
= & 2 \frac{\lambda}{\kappa} \sum_{k} \frac{\sqrt{\kappa} r_{0}\left(\rho_{0}-\sqrt{\kappa} r_{0}\right)}{\left|a_{k}\right|^{2}+\lambda r_{0}^{2}} \\
& -\frac{1}{2}\left\{\frac{1}{\kappa} \sum_{k}\left(\frac{\lambda r_{0}\left(\rho_{0}-\sqrt{\kappa} r_{0}\right)}{\left|a_{k}\right|^{2}+\lambda r_{0}^{2}}\right)^{2}+\sum_{q \neq 0} \frac{1}{\kappa} \sum_{k} \frac{\lambda \bar{\gamma}}{\left|a_{k}\right|^{2}+\lambda r_{0}^{2}} \frac{\lambda \bar{\gamma}}{\left|a_{k-q}\right|^{2}+\lambda r_{0}^{2}} \phi_{q} \phi_{-q}\right. \\
& +\frac{1}{\kappa} \sum_{k}\left(\frac{\lambda r_{0}\left(\rho_{0}-\sqrt{\kappa} r_{0}\right)}{\left|a_{k}\right|^{2}+\lambda r_{0}^{2}}\right)^{2}+\sum_{q \neq 0} \frac{1}{\kappa} \sum_{k} \frac{\lambda \gamma}{|a|^{2}+\lambda r_{0}^{2}} \frac{\lambda \gamma}{\left|a_{k-q}\right|^{2}+\lambda r_{0}^{2}} \bar{\phi}_{q} \bar{\phi}-q \\
& -\frac{\lambda}{\kappa} \sum_{k} \frac{\left|a_{k}\right|^{2}}{\left(\left|a_{k}\right|^{2}+\lambda r_{0}^{2}\right)^{2}}\left(\rho_{0}-\sqrt{\kappa} r_{0}\right)^{2}-\sum_{q \neq 0} \frac{\lambda}{\kappa} \sum_{k} \frac{\bar{a}_{k}}{\left|a_{k}\right|^{2}+\lambda r_{0}^{2}} \frac{a_{k+q}}{\left|a_{k+q}\right|^{2}+\lambda r_{0}^{2}} \rho_{q}^{2} \\
& \left.-\frac{\lambda}{\kappa} \sum_{k} \frac{\left|a_{k}\right|^{2}}{\left(\left|a_{k}\right|^{2}+\lambda r_{0}^{2}\right)^{2}}\left(\rho_{0}-\sqrt{\kappa} r_{0}\right)^{2}-\sum_{q \neq 0} \frac{\lambda}{\kappa} \sum_{k} \frac{a_{k}}{\left|a_{k}\right|^{2}+\lambda r_{0}^{2}} \frac{\bar{a}_{k-q}}{\left|a_{k-q}\right|^{2}+\lambda r_{0}^{2}} \rho_{q}^{2}\right\}+O\left(\xi^{3}\right)
\end{aligned}
$$

Using the BCS equation (5), $\frac{\lambda}{\kappa} \sum_{k} \frac{1}{\left|a_{k}\right|^{2}+\lambda r_{0}^{2}}=1$ and abbreviating

$$
E_{k}^{2}:=\left|a_{k}\right|^{2}+\lambda r_{0}^{2}=k_{0}^{2}+e_{\mathbf{k}}^{2}+\lambda r_{0}^{2}
$$

this becomes

$$
\begin{aligned}
2 \sqrt{\kappa} & r_{0}\left(\rho_{0}-\sqrt{\kappa} r_{0}\right) \cdot 1-\left(\rho_{0}-\sqrt{\kappa} r_{0}\right)^{2} \frac{\lambda}{\kappa} \sum_{k} \frac{\lambda r_{0}^{2}-\left|a_{k}\right|^{2}}{E_{k}^{4}}+\sum_{q \neq 0} \rho_{q}^{2}\left\{\frac{\lambda}{\kappa} \sum_{k} \frac{\bar{a}_{k} a_{k-q}}{E_{k}^{2} E_{k-q}^{2}}\right\} \\
& -\sum_{q \neq 0} \operatorname{Re}\left(e^{-2 i \theta_{0}} \phi_{q} \phi_{-q}\right)\left\{\frac{\lambda}{\kappa} \sum_{k} \frac{\lambda r_{0}^{2}}{E_{k}^{2} E_{k-q}^{2}}\right\} \\
= & 2 \sqrt{\kappa} r_{0}\left(\rho_{0}-\sqrt{\kappa} r_{0}\right) \cdot 1+\left(\rho_{0}-\sqrt{\kappa} r_{0}\right)^{2}-\left(\rho_{0}-\sqrt{\kappa} r_{0}\right)^{2} 2 \frac{\lambda}{\kappa} \sum_{k} \frac{\lambda r_{0}^{2}}{E_{k}^{4}}
\end{aligned}
$$




$$
\begin{aligned}
& +\sum_{q \neq 0} \rho_{q}^{2}\left\{\frac{\lambda}{\kappa} \sum_{k} \frac{\bar{a}_{k} a_{k-q}}{E_{k}^{2} E_{k-q}^{2}}\right\}-\sum_{q \neq 0} \operatorname{Re}\left(e^{-2 i \theta_{0}} \phi_{q} \phi_{-q}\right)\left\{\frac{\lambda}{\kappa} \sum_{k} \frac{\lambda r_{0}^{2}}{E_{k}^{2} E_{k-q}^{2}}\right\} \\
= & \rho_{0}^{2}-\kappa r_{0}^{2}-\left(\rho_{0}-\sqrt{\kappa} r_{0}\right)^{2} 2 \frac{\lambda}{\kappa} \sum_{k} \frac{\lambda r_{0}^{2}}{E_{k}^{4}} \\
& +\sum_{q \neq 0} \rho_{q}^{2}\left\{\frac{\lambda}{\kappa} \sum_{k} \frac{\bar{a}_{k} a_{k-q}}{E_{k}^{2} E_{k-q}^{2}}\right\}-\sum_{q \neq 0} \operatorname{Re}\left(e^{-2 i \theta_{0}} \phi_{q} \phi_{-q}\right)\left\{\frac{\lambda}{\kappa} \sum_{k} \frac{\lambda r_{0}^{2}}{E_{k}^{2} E_{k-q}^{2}}\right\}
\end{aligned}
$$

Therefore one obtains, recalling that $\xi_{k, p}=\frac{1}{\sqrt{\kappa}} \phi_{k-p}-\gamma \delta_{k, p}$,

$$
\begin{aligned}
V\left(\left\{\phi_{q}\right\}\right)- & V\left(\left\{\sqrt{\kappa} \delta_{q, 0} r_{0} e^{i \theta_{0}}\right\}\right)=\sum_{q} \rho_{q}^{2}-\kappa r_{0}^{2}-\log \operatorname{det}\left[I d+\left(\begin{array}{cc}
\lambda \frac{\bar{\gamma}}{|a|^{2}+\lambda r_{0}^{2}} \xi & i g \frac{\bar{A}}{|a|^{2}+\lambda r_{0}^{2}} \xi^{*} \\
i g \frac{A}{|a|^{2}+\lambda r_{0}^{2}} \xi & \lambda \frac{\gamma}{|a|^{2}+\lambda r_{0}^{2}} \xi^{*}
\end{array}\right)\right] \\
= & \sum_{q \neq 0} \rho_{q}^{2}+\left(\rho_{0}-\sqrt{\kappa} r_{0}\right)^{2} 2 \frac{\lambda}{\kappa} \sum_{k} \frac{\lambda r_{0}^{2}}{E_{k}^{4}}-\sum_{q \neq 0} \rho_{q}^{2}\left\{\frac{\lambda}{\kappa} \sum_{k} \frac{\bar{a}_{k} a_{k-q}}{E_{k}^{2} E_{k-q}^{2}}\right\} \\
& +\sum_{q \neq 0} \operatorname{Re}\left(e^{-2 i \theta_{0}} \phi_{q} \phi_{-q}\right)\left\{\frac{\lambda}{\kappa} \sum_{k} \frac{\lambda r_{0}^{2}}{E_{k}^{2} E_{k-q}^{2}}\right\}
\end{aligned}
$$

Consider the coefficient of $\sum_{q \neq 0} \rho_{q}^{2}$. It is given by

$$
\begin{aligned}
1 & -\frac{\lambda}{\kappa} \sum_{k} \frac{\bar{a}_{k} a_{k-q}}{E_{k}^{2} E_{k-q}^{2}}=\frac{1}{2}(1+1)-\frac{1}{2} \frac{\lambda}{\kappa} \sum_{k} \frac{2 \bar{a}_{k} a_{k-q}}{E_{k}^{2} E_{k-q}^{2}} \\
& =\frac{1}{2}\left(\frac{\lambda}{\kappa} \sum_{k} \frac{\left|a_{k}\right|^{2}+\lambda r_{0}^{2}}{E_{k}^{2} E_{k-q}^{2}}+\frac{\lambda}{\kappa} \sum_{k} \frac{\left|a_{k-q}\right|^{2}+\lambda r_{0}^{2}}{E_{k}^{2} E_{k-q}^{2}}\right)-\frac{1}{2} \frac{\lambda}{\kappa} \sum_{k} \frac{2 \bar{a}_{k} a_{k-q}}{E_{k}^{2} E_{k-q}^{2}} \\
& =\frac{1}{2} \frac{\lambda}{\kappa} \sum_{k} \frac{a_{k} \bar{a}_{k}-a_{k} \bar{a}_{k-q}-\bar{a}_{k} a_{k-q}+a_{k-q} \bar{a}_{k-q}}{E_{k}^{2} E_{k-q}^{2}}+\frac{\lambda}{\kappa} \sum_{k} \frac{\lambda r_{0}^{2}}{E_{k}^{2} E_{k-q}^{2}}-\frac{1}{2} \frac{\lambda}{\kappa} \sum_{k} \frac{\bar{a}_{k} a_{k-q}-a_{k} \bar{a}_{k-q}}{E_{k}^{2} E_{k-q}^{2}} \\
& =\frac{1}{2} \frac{\lambda}{\kappa} \sum_{k} \frac{\left(a_{k}-a_{k-q}\right)\left(\bar{a}_{k}-\bar{a}_{k-q}\right)}{E_{k}^{2} E_{k-q}^{2}}+\frac{\lambda}{\kappa} \sum_{k} \frac{\lambda r_{0}^{2}}{E_{k}^{2} E_{k-q}^{2}}-i \frac{\lambda}{\kappa} \sum_{k} \frac{\operatorname{Im}\left(\bar{a}_{k} a_{k-q}\right)}{E_{k}^{2} E_{k-q}^{2}} \\
& =\frac{1}{2} \frac{\lambda}{\kappa} \sum_{k} \frac{q_{0}^{2}+\left(e_{\mathbf{k}}-e_{\mathbf{k}-\mathbf{q}}\right)^{2}}{E_{k}^{2} E_{k-q}^{2}}+\frac{\lambda}{\kappa} \sum_{k} \frac{\lambda r_{0}^{2}}{E_{k}^{2} E_{k-q}^{2}}-i \frac{\lambda}{\kappa} \sum_{k} \frac{k_{0} e_{\mathbf{k}-\mathbf{q}}-\left(k_{0}-q_{0}\right) e_{\mathbf{k}}}{E_{k}^{2} E_{k-q}^{2}} \\
& =\alpha_{q}+i \gamma_{q}+\beta_{q}
\end{aligned}
$$

Inserting (54) in (53), one gets

$$
\begin{aligned}
V\left(\left\{\phi_{q}\right\}\right)- & V\left(\left\{\sqrt{\kappa} \delta_{q, 0} r_{0} e^{i \theta_{0}}\right\}\right)=\left(\rho_{0}-\sqrt{\kappa} r_{0}\right)^{2} 2 \beta_{0}+\sum_{q \neq 0} \rho_{q}^{2}\left\{1-\frac{\lambda}{\kappa} \sum_{k} \frac{2 \bar{a}_{k} a_{k-q}}{E_{k}^{2} E_{k-q}^{2}}\right\} \\
& +\sum_{q \neq 0} \operatorname{Re}\left(e^{-2 i \theta_{0}} \phi_{q} \phi_{-q}\right)\left\{\frac{\lambda}{\kappa} \sum_{k} \frac{\lambda r_{0}^{2}}{E_{k}^{2} E_{k-q}^{2}}\right\} \\
= & \left(\rho_{0}-\sqrt{\kappa} r_{0}\right)^{2} 2 \beta_{0}+\sum_{q \neq 0} \rho_{q}^{2}\left(\alpha_{q}+i \gamma_{q}\right) \\
& +\sum_{q \neq 0} \rho_{q}^{2} \beta_{q}+\sum_{q \neq 0} \frac{e^{-2 i \theta_{0}} \phi_{q} \phi_{-q}+e^{2 i \theta_{0}} \bar{\phi}_{q} \bar{\phi}_{-q}}{2} \beta_{q}
\end{aligned}
$$


Since $\beta_{q}=\beta_{-q}$, the last two q-sums in (55) may be combined to give

$$
\begin{aligned}
\sum_{q \neq 0} & \frac{\rho_{q}^{2}+\rho_{-q}^{2}}{2} \beta_{q}+\sum_{q \neq 0} \frac{e^{-2 i \theta_{0}} \phi_{q} \phi_{-q}+e^{2 i \theta_{0}} \bar{\phi}_{q} \bar{\phi}_{-q}}{2} \beta_{q} \\
& =\frac{1}{2} \sum_{q \neq 0}\left(\phi_{q} \bar{\phi}_{q}+\phi_{-q} \bar{\phi}_{-q}+e^{-2 i \theta_{0}} \phi_{q} \phi_{-q}+e^{2 i \theta_{0}} \bar{\phi}_{q} \bar{\phi}_{-q}\right) \beta_{q} \\
& =\frac{1}{2} \sum_{q \neq 0}\left(e^{-i \theta_{0}} \phi_{q}+e^{i \theta_{0}} \bar{\phi}_{-q}\right)\left(e^{i \theta_{0}} \bar{\phi}_{q}+e^{-i \theta_{0}} \phi_{-q}\right) \beta_{q} \\
& =\frac{1}{2} \sum_{q \neq 0}\left|e^{-i \theta_{0}} \phi_{q}+e^{i \theta_{0}} \bar{\phi}_{-q}\right|^{2} \beta_{q}
\end{aligned}
$$

This proves Theorem $2 \square$

\section{The Effective Potential with a U(1) Symmetry Breaking External Field}

We consider now the situation where a small external field is added to the action which breaks the $U(1)$ symmetry. In that case, the partition function (13) changes to

$$
Z_{r}=\int e^{\frac{\lambda}{(\kappa)^{3}} \sum_{k, p, q} \bar{\psi}_{k \uparrow} \bar{\psi}_{q-k \downarrow} \psi_{q-p \downarrow} \psi_{p \uparrow}+\frac{1}{\kappa} \sum_{k}\left[r \psi_{k \uparrow} \psi_{-k \downarrow}+\bar{r} \bar{\psi}_{-k \downarrow} \bar{\psi}_{k \uparrow}\right]} d \mu_{C}
$$

After a Hubbard-Stratonovich transformation, this becomes

$$
Z_{r}=\int e^{-V_{r}\left(\left\{\phi_{q}\right\}\right)} \prod_{q} \frac{d \phi_{q} d \bar{\phi}_{q}}{\pi}
$$

where (recall that $\kappa:=\beta L^{d}$ )

$$
V_{r}\left(\left\{\phi_{q}\right\}\right)=\sum_{q}\left|\phi_{q}\right|^{2}-\log \operatorname{det}\left[\begin{array}{cc}
I d & C\left(\frac{i g \phi^{*}}{\sqrt{\kappa}}-\bar{r} \delta_{k, p}\right) \\
\bar{C}\left(\frac{i g \phi}{\sqrt{\kappa}}+r \delta_{k, p}\right) & I d
\end{array}\right]
$$

For the, say, $\left\langle\bar{\psi}_{\sigma} \psi_{\sigma}\right\rangle$ and $\left\langle\psi_{\uparrow} \psi_{\downarrow}\right\rangle$ correlations one obtains similarly [5]:

$$
\begin{aligned}
\left\langle\bar{\psi}_{k \uparrow} \psi_{k \uparrow}\right\rangle_{r} & =\kappa\left\langle F_{r}(k)\right\rangle_{r} \\
\left\langle\psi_{k \uparrow} \psi_{-k \downarrow}\right\rangle_{r} & =\kappa\left\langle G_{r}(k)\right\rangle_{r}
\end{aligned}
$$

where

$$
\begin{gathered}
\left.F_{r}(k)=F_{r}(k ; \phi)=\left[\begin{array}{cc}
a_{s} \delta_{s, t} & \left(\frac{i g \bar{\phi}_{t-s}}{\sqrt{\kappa}}-\bar{r} \delta_{s, t}\right) \\
\left(\frac{i g \phi_{s-t}}{\sqrt{\kappa}}+r \delta_{s, t}\right) & a_{-s} \delta_{s, t}
\end{array}\right)_{s, t}\right]_{k \uparrow, k \uparrow}^{-1} \\
\left.G_{r}(k)=G_{r}(k ; \phi)=\left[\begin{array}{cc}
a_{s} \delta_{s, t} & \left(\frac{i g \bar{\phi}_{t-s}}{\sqrt{\kappa}}-\bar{r} \delta_{s, t}\right) \\
\left(\frac{i g \phi_{s-t}}{\sqrt{\kappa}}+r \delta_{s, t}\right) & a_{-s} \delta_{s, t}
\end{array}\right)_{s, t}^{-1}\right]_{k \downarrow, k \uparrow}^{-1}
\end{gathered}
$$


and in $(60,61)$ the expectation on the left is given by the Grassmann integral with external field and the expectation on the right is given by $\langle F\rangle_{r}=\int F(\phi) e^{-V_{r}(\phi)} / \int e^{-V_{r}(\phi)}$.

In (59-63), the external field $r$ only shows up in conjunction with the $\phi_{0}$ variable through the combination $\frac{\phi_{0}}{\sqrt{\kappa}}-i \frac{r}{g}$. By substitution of variables one has, if $\phi_{0}=u_{0}+i v_{0}$ and $r=|r| e^{i \alpha}$

$$
\begin{gathered}
\int_{\mathbf{R}^{2}} f\left(\frac{\phi_{0}}{\sqrt{\kappa}}-i \frac{r}{g}, \frac{\bar{\phi}_{0}}{\sqrt{\kappa}}+i \frac{\bar{r}}{g}\right) e^{-\left(u_{0}^{2}+v_{0}^{2}\right)} d u_{0} d v_{0}= \\
\int_{\mathbf{R}^{2}} f\left(e^{i \alpha} \frac{\phi_{0}}{\sqrt{\kappa}}, e^{-i \alpha} \frac{\bar{\phi}_{0}}{\sqrt{\kappa}}\right) e^{-\left(u_{0}^{2}+\left(v_{0}+\sqrt{\kappa} \frac{|r|}{g}\right)^{2}\right)} d u_{0} d v_{0}
\end{gathered}
$$

Thus we can write

$$
Z_{r}=\int e^{-U_{r}\left(\left\{\phi_{q}\right\}\right)} \prod_{q} \frac{d \phi_{q} d \bar{\phi}_{q}}{\pi}
$$

where

$$
U_{r}\left(\left\{\phi_{q}\right\}\right)=u_{0}^{2}+\left(v_{0}+\sqrt{\kappa} \frac{|r|}{g}\right)^{2}+\sum_{q \neq 0}\left|\phi_{q}\right|^{2}-\log \operatorname{det}\left[\begin{array}{cc}
I d & \frac{i g}{\sqrt{\kappa}} C \tilde{\phi}^{*} \\
\frac{i g}{\sqrt{\kappa}} \bar{C} \tilde{\phi} & I d
\end{array}\right]
$$

and

$$
\tilde{\phi}=\left(\tilde{\phi}_{k-p}\right)_{k, p}, \quad \tilde{\phi}_{q}= \begin{cases}\phi_{q} & \text { if } q \neq 0 \\ e^{i \alpha} \phi_{0} & \text { if } q=0\end{cases}
$$

Furthermore

$$
\begin{aligned}
\left\langle\bar{\psi}_{k \uparrow} \psi_{k \uparrow}\right\rangle_{r} & =\kappa\left\langle\tilde{F}_{0}(k)\right\rangle_{U_{r}} \\
\left\langle\psi_{k \uparrow} \psi_{-k \downarrow}\right\rangle_{r} & =\kappa\left\langle\tilde{G}_{0}(k)\right\rangle_{U_{r}}
\end{aligned}
$$

where $\tilde{F}_{0}(k)$ and $\tilde{G}_{0}(k)$ are given by $(62,63)$ with $r=0$ and $\phi$ substituted by $\tilde{\phi}$. The expectations on the right hand side of $(68,69)$ are now taken with respect to $U_{r}$, that is $\langle F\rangle_{U_{r}}=\int F(\phi) e^{-U_{r}(\phi)} / \int e^{-U_{r}(\phi)}$.

Thus in the case with a small external field we would ask for the global minimum of $U_{r}$ and for the second order Taylor expansion around it. One has the following

Corollary: Let $U_{r}$ be the effective potential (66) with a small external $U(1)$ symmetry breaking field $r=|r| e^{i \alpha}$. Let $\tilde{\phi}$ be given by (6r). Then:

(i) The global minimum of

$$
\operatorname{Re} U_{r}\left(\left\{\phi_{q}\right\}\right)=u_{0}^{2}+\left(v_{0}+\sqrt{\kappa} \frac{|r|}{g}\right)^{2}+\sum_{q \neq 0}\left|\phi_{q}\right|^{2}-\log \left|\operatorname{det}\left[\begin{array}{cc}
I d & \frac{i g}{\sqrt{\kappa}} C \tilde{\phi}^{*} \\
\frac{i g}{\sqrt{\kappa}} \bar{C} \tilde{\phi} & I d
\end{array}\right]\right|
$$


is unique and is given by $\phi_{q}^{\min }=\delta_{q, 0} \sqrt{\kappa}$ i $y_{0}$ where $y_{0}=y_{0}(|r|)$ is the unique global minimum of the function $V_{\mathrm{BCS}, r}: \mathbf{R} \rightarrow \mathbf{R}$,

$$
\begin{aligned}
V_{\mathrm{BCS}, r}(y) & :=U_{r}\left(u_{0}=0, v_{0}=\sqrt{\kappa} y ; \phi_{q}=0 \text { for } q \neq 0\right) \\
& =\kappa\left\{\left(y+\frac{|r|}{g}\right)^{2}-\frac{1}{\kappa} \sum_{k} \log \left[1+\frac{\lambda y^{2}}{k_{0}^{2}+e_{\mathbf{k}}^{2}}\right]\right\} .
\end{aligned}
$$

(ii) The second order Taylor expansion of $U_{r}$ around $\phi^{\text {min }}$ is given by

$$
\begin{gathered}
U_{r}\left(\left\{\phi_{q}\right\}\right)=U_{r, \min }+2 \beta_{0}\left(v_{0}-\sqrt{\kappa} y_{0}\right)^{2}+\sum_{q \neq 0}\left(\alpha_{q}+i \gamma_{q}\right)\left|\phi_{q}\right|^{2}+\frac{1}{2} \sum_{q \neq 0} \beta_{q}\left|e^{-i \alpha} \phi_{q}-e^{i \alpha} \bar{\phi}_{-q}\right|^{2} \\
+\frac{|r|}{g\left|y_{0}\right|}\left(u_{0}^{2}+\left(v_{0}-\sqrt{\kappa} y_{0}\right)^{2}+\sum_{q \neq 0}\left|\phi_{q}\right|^{2}\right)+O\left(\left(\phi-\phi^{\min }\right)^{3}\right) .
\end{gathered}
$$

where $U_{r, \min }:=U_{r}\left(\left\{\phi_{q}^{\min }\right\}\right)$ and the coefficients $\alpha_{q}, \beta_{q}$ and $\gamma_{q}$ are given by (42) of Theorem 2 but $E_{k}$ in this case is given by $E_{k}^{2}=\left|a_{k}\right|^{2}+\lambda y_{0}^{2}=k_{0}^{2}+e_{\mathbf{k}}^{2}+\lambda y_{0}^{2}$.

Remark: Of course one has $\lim _{|r| \rightarrow 0} \lambda y_{0}(|r|)^{2}=\lambda r_{0}^{2}=\Delta^{2}$ where $\pm r_{0}$ is the global minimum of $V_{\mathrm{BCS}, r=0}$.

Proof: (i) As in the proof of Theorem I one shows that

$$
\begin{aligned}
\log \left|\operatorname{det}\left[\begin{array}{cc}
I d & \frac{i g}{\sqrt{\kappa}} C \tilde{\phi}^{*} \\
\frac{i g}{\sqrt{\kappa}} \bar{C} \tilde{\phi} & I d
\end{array}\right]\right| & \leq \sum_{k} \log \left[1+\frac{\frac{\lambda}{\kappa} \sum_{q}\left|\phi_{q}\right|^{2}}{\left|a_{k}\right|^{2}}\right] \\
& =: \sum_{k} \log \left[1+\frac{\lambda\left(x^{2}+y^{2}\right)}{\left|a_{k}\right|^{2}}\right]
\end{aligned}
$$

where we abbreviated

$$
x^{2}:=\frac{1}{\kappa}\left(u_{0}^{2}+\sum_{q \neq 0}\left|\phi_{q}\right|^{2}\right), \quad y^{2}:=\frac{1}{\kappa} v_{0}^{2}
$$

Thus

$$
\begin{aligned}
\operatorname{Re} U_{r}\left(\left\{\phi_{q}\right\}\right) & \geq\left(v_{0}+\sqrt{\kappa} \frac{|r|}{g}\right)^{2}+u_{0}^{2}+\sum_{q \neq 0}\left|\phi_{q}\right|^{2}-\sum_{k} \log \left[1+\frac{\lambda\left(x^{2}+y^{2}\right)}{\left|a_{k}\right|^{2}}\right] \\
& =: \kappa W_{r}(x, y)
\end{aligned}
$$

where

$$
W_{r}(x, y)=x^{2}+\left(y+\frac{|r|}{g}\right)^{2}-\frac{1}{\kappa} \sum_{k} \log \left[1+\frac{\lambda\left(x^{2}+y^{2}\right)}{\left|a_{k}\right|^{2}}\right]
$$


The global minimum of $W_{r}$ is unique and given by $x=0$ and $y=y_{0}$ where $y_{0}$ is the unique global minimum of (70). Since $U_{r}\left(u_{0}=0, v_{0}=\sqrt{\kappa} y ; \phi_{q}=0\right.$ for $\left.q \neq 0\right)=V_{\mathrm{BCS}, r}(y)$, part (i) follows.

(ii) Part (ii) is proven in the same way as Theorem II. One has

$$
\begin{aligned}
U_{r}\left(\left\{\phi_{q}\right\}\right)- & U_{r, \min }=u_{0}^{2}+\sum_{q \neq 0}\left|\phi_{q}\right|^{2}+\left(v_{0}+\sqrt{\kappa} \frac{|r|}{g}\right)^{2}-\left(\sqrt{\kappa} y_{0}+\sqrt{\kappa} \frac{|r|}{g}\right)^{2} \\
& -\log \left\{\operatorname{det}\left[\begin{array}{cc}
a_{k} \delta_{k, p} & \frac{i g}{\sqrt{\kappa}} \overline{\tilde{\phi}}_{p-k} \\
\frac{i g}{\sqrt{\kappa}} \tilde{\phi}_{k-p} & a_{-k} \delta_{k, p}
\end{array}\right] / \operatorname{det}\left[\begin{array}{cc}
a_{k} \delta_{k, p} & \frac{i g}{\sqrt{\kappa}} e^{-i \alpha}(-i) y_{0} \delta_{k, p} \\
\frac{i g}{\sqrt{\kappa}} e^{i \alpha} i y_{0} \delta_{k, p} & a_{-k} \delta_{k, p}
\end{array}\right]\right\} \\
= & u_{0}^{2}+\sum_{q \neq 0}\left|\phi_{q}\right|^{2}+\left(v_{0}-\sqrt{\kappa} y_{0}\right)^{2}+2\left(v_{0}-\sqrt{\kappa} y_{0}\right)\left(\sqrt{\kappa} y_{0}+\sqrt{\kappa} \frac{|r|}{g}\right) \\
& -\log \operatorname{det}\left[I d+\left(\begin{array}{cc}
a_{k} \delta_{k, p} & \frac{i g}{\sqrt{\kappa}} e^{-i \alpha}(-i) y_{0} \delta_{k, p} \\
\frac{i g}{\sqrt{\kappa}} e^{i \alpha} i y_{0} \delta_{k, p} & a_{-k} \delta_{k, p}
\end{array}\right)^{-1}\left(\begin{array}{cc}
0 & \frac{i g}{\sqrt{\kappa}} \bar{\xi}_{p-k} \\
\frac{i g}{\sqrt{\kappa}} \xi_{k-p} & 0
\end{array}\right)\right]
\end{aligned}
$$

where in this case

$$
\xi_{k-p}:=\tilde{\phi}_{k-p}-\sqrt{\kappa} e^{i \alpha} i y_{0} \delta_{k, p}
$$

The expression $\log \operatorname{det}[I d+\cdots]$ is expanded as in the proof of Theorem II. One obtains, if $E_{k}^{2}:=\left|a_{k}\right|^{2}+\lambda y_{0}^{2}$,

$$
\begin{aligned}
\log \operatorname{det}[I d+\cdots]=2 & \sqrt{\kappa} y_{0}\left(v_{0}-\sqrt{\kappa} y_{0}\right) \frac{\lambda}{\kappa} \sum_{k} \frac{1}{E_{k}^{2}} \\
& +\frac{\lambda}{\kappa} \sum_{k} \frac{\lambda y_{0}^{2}\left(u_{0}^{2}-\left(v_{0}-\sqrt{\kappa} y_{0}\right)^{2}\right)}{E_{k}^{4}}+\frac{\lambda}{\kappa} \sum_{k} \frac{\left|a_{k}\right|^{2}\left(u_{0}^{2}+\left(v_{0}-\sqrt{\kappa} y_{0}\right)^{2}\right)}{E_{k}^{4}} \\
& +\frac{1}{2} \frac{\lambda}{\kappa} \sum_{q \neq 0} \sum_{k}\left(\frac{a_{k} \bar{a}_{k-q}}{E_{k}^{2} E_{k-q}^{2}}+\frac{\bar{a}_{k} a_{k+q}}{E_{k}^{2} E_{k+q}^{2}}\right)\left|\phi_{q}\right|^{2} \\
& -\frac{1}{2} \frac{\lambda}{\kappa} \sum_{q \neq 0} \sum_{k}\left(\frac{\lambda y_{0}^{2} e^{2 i \alpha}}{E_{k}^{2} E_{k+q}^{2}} \bar{\phi}_{q} \bar{\phi}_{-q}+\frac{\lambda y_{0}^{2} e^{-2 i \alpha}}{E_{k}^{2} E_{k-q}^{2}} \phi_{q} \phi_{-q}\right)
\end{aligned}
$$

Since $y_{0}$ is a minimum of $V_{\mathrm{BCS}, r}$, one has the BCS equation

$$
2\left(y_{0}+\frac{|r|}{g}\right)-\frac{\lambda}{\kappa} \sum_{k} \frac{2 y_{0}}{E_{k}^{2}}=0 \text { or } \quad \frac{\lambda}{\kappa} \sum_{k} \frac{1}{E_{k}^{2}}=1-\frac{|r|}{g\left|y_{0}\right|}
$$

Using this, one gets (observe that $y_{0}$ is negative)

$$
\begin{aligned}
U_{r}\left(\left\{\phi_{q}\right\}\right)= & U_{r, \min }+\frac{|r|}{g\left|y_{0}\right|}\left(u_{0}^{2}+\left(v_{0}-\sqrt{\kappa} y_{0}\right)^{2}\right)+2 \frac{\lambda}{\kappa} \sum_{k} \frac{\lambda y_{0}^{2}\left(v_{0}-\sqrt{\kappa} y_{0}\right)^{2}}{E_{k}^{4}} \\
& +\sum_{q \neq 0}\left\{1-\frac{\lambda}{\kappa} \sum_{k} \frac{\bar{a}_{k} a_{k-q}}{E_{k}^{2} E_{k-q}^{2}}\right\}\left|\phi_{q}\right|^{2}+\frac{1}{2} \frac{\lambda}{\kappa} \sum_{q \neq 0} \sum_{k}\left(\frac{\lambda y_{0}^{2} e^{2 i \alpha}}{E_{k}^{2} E_{k+q}^{2}} \bar{\phi}_{q} \bar{\phi}_{-q}+\frac{\lambda y_{0}^{2} e^{-2 i \alpha}}{E_{k}^{2} E_{k-q}^{2}} \phi_{q} \phi_{-q}\right)
\end{aligned}
$$


Using the BCS equation (79) again, one obtains (compare (54))

$$
1-\frac{\lambda}{\kappa} \sum_{k} \frac{\bar{a}_{k} a_{k-q}}{E_{k}^{2} E_{k-q}^{2}}=\alpha_{q}+i \gamma_{q}+\beta_{q}+\frac{|r|}{g\left|y_{0}\right|}
$$

Substituting this in (80) and rearranging as in the proof of Theorem II proves part (ii)

\section{References}

[1] J. Feldman, J. Magnen, V. Rivasseau, E. Trubowitz, Fermionic Many Body Models, in: CRM Proceedings and Lecture Notes Vol. 7, Mathematical Quantum Theory I: Field Theory and Many Body Theory, eds. J. Feldman, R. Froese, L. M. Rosen, 1994.

[2] J. Feldman, J. Magnen, V. Rivasseau, E. Trubowitz, Ward Identities and a Perturbative Analysis of a U(1) Goldstone Boson in a Many Fermion System, Helvetia Physica Acta 66, 1993, 498-550.

[3] T. Chen, J. Fröhlich, M. Seifert, Renormalization Group Methods: Landau-Fermi Liquid and BCS Superconductor, Proceedings of the Les Houches session Fluctuating Geometries in Statistical Mechanics and Field Theory, eds. F. David, P. Ginsparg, J. Zinn-Justin, 1994.

[4] J. Feldman, E. Trubowitz, Perturbation Theory for Many Fermion Systems, Helv. Phys. Acta 63, 1990, 156-260; The Flow of an Electron Phonon System to the Superconducting State, Helv. Phys. Acta 64, 1991, 214-357.

[5] D. Lehmann, The Many-Electron System in the Forward, Exchange and BCS Approximation, Comm. Math. Phys. 198, 427-468, 1998. 